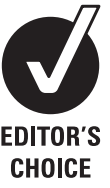

10.1136/ebm1049

Department of Pediatric and Adolescent Medicine, Mayo Clinic, Rochester, Minnesota, USA

Correspondence to: Philip R Fischer Department of Pediatric and Adolescent Medicine, Mayo Clinic, 200 First Street SW, Rochester, MN 55905, USA; fischer.phil@mayo.edu
Randomised controlled trial

\section{Prophylactic paracetamol at the time of infant vaccination reduces the risk of fever but also reduces antibody response}

\section{Jason H Homme, Philip R Fischer}

\author{
Commentary on: Prymula R, Siegrist CA, Chlibek R, et al. Effect of prophylactic paracetamol \\ administration at time of vaccination on febrile reactions and antibody responses in children: two \\ open-label, randomised controlled trials. Lancet 2009;374:1339-50.
}

Although immunisation is one of the great accomplishments of the field of public health ${ }^{1}$ and routine vaccination is extremely beneficial for children, families and care providers are well aware that bothersome side effects of immunisation are common. For many providers, it is common practice to suggest that an antipyretic be given preventively at the time of vaccine administration ${ }^{2}$ - even though the value of this practice has not been fully proven. ${ }^{3}$ Prymula and colleagues compared postvaccine fever and postvaccine changes in vaccine-specific antibody titres in children who either did or did not receive scheduled paracetamol with immunisations.

At 10 centres in the Czech Republic, 459 healthy infants undergoing initial (9-16 weeks of age) and booster (12-15 months of age) vaccinations (oral rotavirus vaccine, and intramuscular 10 -valent pneumococcal vaccine and combined diphtheria, tetanus, acellular pertussis, polio, Haemophilus influenzae type $b$ and hepatitis $B$ vaccine) were randomised to receive or not to receive prophylactic paracetamol (at the time of vaccination and 6-8 hourly during the subsequent $24 \mathrm{~h}, 34-54 \mathrm{mg} / \mathrm{kg} /$ day) via rectal suppository. Fever (defined as rectal temperature $\geq 38.0^{\circ} \mathrm{C}$ ) and antibody titres were followed.

Fever was significantly less common in paracetamoltreated children after initial (42\% vs 66\%) and booster (36\% vs 58\%) vaccination. Children who received paracetamol had significantly lower antibody titres to each vaccine-related antigen. The antibody titres were not statistically related to whether the child had fever but were related to whether the child received paracetamol. Nonetheless, more than $96 \%$ of children achieved antibody titres considered 'protective' whether or not they had received prophylactic paracetamol. Thus, presymptomatic paracetamol given at the time of routine infant immunisation prevents some mild fevers, maintains rates of immune protection and reduces resulting postvaccine antibody titres.

The patient selection and vaccination procedures were reasonable and generally representative of routine paediatric care around the world. The study was not blinded, but objective outcomes (temperature, antibody levels) were probably not biased by the lack of blinding. Comparative data were adequate to differentiate intervention effects (antibody level) from a confounding outcome effect (fever). Sample size was adequate to power the intended analyses appropriately. Statistical techniques were appropriate. No significant conclusion-altering biases were detected. All interpretations presented by the authors seemed to be fully in line with the data presented.

Now that paracetamol use is known to be associated with decreased postvaccine antibody levels, practitioners must balance paracetamol's benefit (more comfort) against its risk (lower antibody titres). One of the authors of this commentary never liked to give presymptomatic paracetamol and now is even more resolute in that habit. The other is in a practice group that has, on the basis of this study, chosen to advise paracetamol use only if postvaccine symptoms develop. Another commentator now suggests that it "seems reasonable to reconsider ... the routine use of paracetamol for paediatric immunisations."4 Meanwhile, the American Academy of Pediatrics continues to say that this study "should not prevent the use of antipyretics either prophylactically or therapeutically". ${ }^{5}$

Prymula and colleagues confirm that peri-vaccination paracetamol is sometimes associated with less fever and discomfort. ${ }^{6}$ Their results are new, however, with regard to antibody responses. Previously, antibody responses to diphtheria-tetanus-pertussis vaccine were not altered by paracetamol, though details of the timing and dosing of paracetamol were not presented. ${ }^{7}$ When paracetamol was 
given $4 \mathrm{~h}$ after vaccination in a study involving wholecell pertussis vaccine and just 59 children ( 25 control, 34 treated), antibody titres were not changed by the use of paracetamol. ${ }^{8}$ Adults receiving flu vaccine achieve "protective' antibody levels regardless of whether they use paracetamol around the time of vaccination. ${ }^{9}$

Future studies should assess how timing (with or after vaccination), dosing $(\mathrm{mg} / \mathrm{kg}$ ) and administration (rectal or oral) of paracetamol relate to antibody responses. The effects of the child's age on the deleterious effect of paracetamol and the impact of ibuprofen on immune responsiveness could also be studied. It is currently unknown how various vaccine combinations might modify the paracetamol effect.

\section{Competing interests None.}

\section{References}

1. Jacobson RM. An update on childhood and adolescent vaccines. Mayo Clin Proc 2009;84:457-9; quiz 460.
2. Centers for Disease Control and Prevention. DiphtheriaTetanus-Pertussis Vaccine Information Statement. http://www. immunize.org/VIS/dtap01.pdf (Accessed 11 February 2010).

3. Manley J, Taddio A. Acetaminophen and ibuprofen for prevention of adverse reactions associated with childhood immunization. Ann Pharmacother 2007;41:1227-32.

4. Bell EA. Rethinking acetaminophen for immunizations. Infect Dis Children January 2010:14.

5. Brady MT, Swanson JT. More study needed on antipyretics' effect on vaccine responses. AAP News 2010;31:1.

6. Lewis K, Cherry JD, Sachs MH, et al. The effect of prophylactic acetaminophen administration on reactions to DTP vaccination. Am J Dis Child 1988;142:62-5.

7. Long SS, Deforest A, Smith DG, et al. Longitudinal study of adverse reactions following diphtheria-tetanus-pertussis vaccine in infancy. Pediatrics 1990;85:294-302.

8. Uhari M, Hietala J, Viljanen MK. Effect of prophylactic acetaminophen administration on reaction to DTP vaccination. Acta Paediatr Scand 1988;77:747-51.

9. Chernesky M, O'Neill D, Pickard L, et al. Immunogenicity and adverse reactions of influenza vaccination in elderly patients given acetaminophen or placebo. Clin Diagn Virol 1993;1:129-36. 